\title{
Fractal evidences in wind speed time series
}

\author{
Luigi Fortuna and Silvia Nunnari \\ Dipartimento di Ingegneria Elettrica, Elettronica ed Informatica \\ Universita' degli Studi di Catania \\ Viale A. Doria, 6, \\ 95125 Catania, Italy \\ Email: luigi.fortuna,silvia.nunnari@dieei.unict.it
}

\author{
Giorgio Guariso \\ Dipartimento di Elettronica,Informatica e Bioingegneria \\ Politecnico di Milano \\ Via G. Ponzio, 34/5 \\ 20133 Milano, Italy \\ Email: giorgio.guariso@polimi.it
}

\begin{abstract}
This paper describes some evidence of fractal order features in wind speed time series recorded at different observation stations both in USA and in Italy. Analysis were performed by using mono-fractal, multi-fractal and power spectra approaches. Results show that the average value of the box dimension for daily and hourly mean wind speed is $D=1.19$ and $D=1.41$ respectively, thus indicating that this kind of time series are fractal. The estimated average value of the Hurst exponent is $H=0.81$ and $H=0.75$ for daily and hourly time series respectively. From these Hurst exponents it is possible to infer the persistent behavior of wind speed. Furthermore, multi-fractal analysis shows that wind speed exhibits a bell-like shape spectrum with average width $\Delta \alpha=0.47$. Power spectra analysis has pointed out that wind speed time series behave as $1 / f^{\beta}$ noise with average value of the $\beta$ exponent of 0.46 and 1.37 for daily mean and hourly mean time series respectively. These latter results can be interpreted by saying that wind speed time series are Brown noise like.
\end{abstract}

\section{INTRODUCTION}

As widely pointed out by several authors, wind speed is an important resource of renewable energy and a lot of effort has been devoted to study short term prediction models based on time series recorded at different meteo stations. A review on 30 years of history of the wind power short-term prediction was reported in [1], while a more recent and comprehensive review can be found in [2]. Wind models are classified into two broad classes, depending on the fact that a Numerical Weather Prediction model (NWP) is involved or not. Usually models that include NWP are referred to as physical models, while models of the other class are referred to as statistical or time series approaches. Models that make use of NWP are quite complex and considered for long time horizons, while time series approach are more appropriate for short time horizons (less then 24 hours), at specific points. This latter kind of models can be very useful for forecasting the production of wind farms, since the conventional power plants in the electric network require a prediction horizon of 3 to 10 hours, depending on the size and type of production units, to be optimally managed. Despite this great interest, to our knowledge, little effort has been devoted to understanding the deep nature of wind speed time series. Recent papers (see for instance [3]) pointed out that wind speed time series exhibits fractal behaviors. Furthermore [4] presented some results concerning multifractal analysis of wind speed time series. In this paper we investigate the nature of wind speed time series by using several approaches ranging from fractal and multifractal analysis to power spectrum analysis.

\section{SOME BACKGROUND ABOUT FRACTAL AND SPECTRAL ANALYSIS}

Fractals are typically self-similar patterns, where selfsimilar means they are "the same from near as from far" [5]. Strogatz in his book about nonlinear dynamics and chaos [6] describes fractals as complex geometric shapes with fine structure at arbitrarily small scales. In simple words, if we magnify a tiny part of a fractal, we will see features reminiscent of the whole. Sometimes the similarity is exact but more often it is only approximate or statistical. When similarity is exact, they are said to be "scale-free". Formally, a time series $x(t)$ is scale invariant when $x(s t)=s^{-D} x(t)$, where the power index $D$ is the fractal dimension, which usually exceeds its topological dimension. In other terms, while a time series is a line from the topological point of view (i.e. 1-dimensional), it tends to be be more similar to a surface from the fractal point of view, being its dimension a number between 1 and 2 . A practical interpretation of the fractal dimension of a time series is that it reflects the irregularity degree of observed data. The scale-free feature may be interpreted in the sense that when the variable $s$ zooms in or out $\lambda$ times, then the function is enlarged or reduced $\lambda^{-D}$ times, while the shape and features of the original function are leaved unchanged. Particular kinds of time series, originated by complex nonlinear dynamic systems, such as the geophysical ones, can be sometimes conveniently characterized as fractal objects[7]. If the fractal dimension does not change with scale, such kind of fractal is claimed as a mono-fractal or also as single-scale, otherwise it is referred to as multi-fractal. The fractal dimension $D$ obtained by the box counting approach and the Hurst index $H$ can measure the single scale fractal characteristic effectively.

\section{A. Mono-fractal time series analysis}

Roughly speaking mono-fractal analysis of a given time series consists in computing its fractal dimension $D$ by using one of several methods proposed in literature. To this purpose, it is to be observed that while the Hausdorff dimension, based on covering the time series by using small circles of radius $\varepsilon$, has a rigorous mathematical definition, it is not practical from the computation point of view. Thus usually the Hausdorff dimension is substituted by the so-called box dimension defined as

$$
D=-\lim _{\varepsilon \rightarrow 0} \frac{\log N_{\varepsilon}}{\log \varepsilon}
$$

where $\varepsilon$ is a small square lattice with side $\varepsilon$ and $N_{\varepsilon}$ is the number of grids needed to cover the time series. In order 
to compute the box dimension several approaches have been proposed in the literature such as [8] [9]. A useful approach to evaluate the box dimension $D$ of a given time series is to observe that if the relation between $N_{\varepsilon}$ and $\varepsilon$ is a power law of the type

$$
N_{\varepsilon} \propto \varepsilon^{-D}
$$

then by taking the log of both members in expression (2) we have

$$
\log N_{\varepsilon}=\log C-D \log \varepsilon
$$

which represents a straight line in a log-log diagram, drawn in the plane $N_{\varepsilon}$ versus $\varepsilon$. The angular coefficient of this line is $D$ while $C$ is a constant. Thus in order to estimate $D$ it will be enough to approximate the curve $\log N_{\varepsilon}$ versus $\log \varepsilon$ with a regression line by using the traditional least square approach.

Another useful strategy to analyze the mono-fractal nature of time series consists in evaluating the Hurst exponent, originally developed by the hydrologist H.E. Hurst. Later on Mandelbrot, the pioneer of fractal geometry, recognized that the Hurst exponent can be related to the fractal dimension of a time series by the simple expression $D=2-H$ [5]. One of best known method to estimate the Hurst exponent of a time series is probably the R/S analysis proposed by [10] which is summarized below. For a given time series $x_{i}, i=1,2, \ldots, N$, consider its first $n \leq N$ values and compute the average value

$$
\langle x\rangle=\frac{1}{n} \sum_{i=1}^{n} x_{i}
$$

and the standard deviation

$$
S=\sqrt{\frac{1}{n} \sum_{i=1}^{n}\left(x_{i}-\langle x\rangle\right)^{2}}
$$

The so-called $(R / S)_{n}$ statistic is defined as

$$
(R / S)_{n}=\frac{1}{S}\left[\operatorname{Max}_{n} \sum_{i=1}^{n}\left(x_{i}-\langle x\rangle\right)-\operatorname{Min}_{n} \sum_{i=1}^{n}\left(x_{i}-\langle x\rangle\right)\right]
$$

One of the achievements of the $\mathrm{R} / \mathrm{S}$ analysis is that the ratio $(R / S)_{n}$ tends to $C n^{H}$ as $n \rightarrow \infty$, being $C$ a constant and $H$ the Hurst exponent. Thus, since $\ln (R / S)_{n}=\ln C+H \ln (n)$, by approximating the $\ln (R / S)_{n}$ versus $\ln (n)$ graph by a simple regression line, it is possible to estimate $H$ by evaluating its slope. However, others authors recognized that the original R/S analysis may show some problem when the considered time series is not large enough and proposed some improvements. In this paper we consider the procedure developed by [11], who also performs the so-called corrected Empirical and Theoretical R/S analysis. Practical examples will be shown in section III. To conclude this short introduction about the Hurst exponent, we remind here that it is used as a measure of long term memory of time series. It relates to the autocorrelations of the time series, and the rate at which this decreases as the lag between pairs of values increases. A value $H$ in the range $0.5<H<1$ indicates a time series with long- term positive autocorrelation, meaning both that a high value in the series will probably be followed by another high value and that the values a long time into the future will also tend to be high. A value in the range $0<H<0.5$ indicates a time series with long term switching between high and low values in adjacent pairs, meaning that a single high value will probably be followed by a low value and that the value after will tend to be high, with this tendency to switch between high and low values lasting a long time into the future. Finally, a value of $H=0.5$ can indicate a completely uncorrelated series.

\section{B. Multi-fractal time series analysis}

If the fractal dimension does not change with scale, the time series is said be a single scale fractal, while if the fractal dimension varies with scale, i.e. as a function of $s$, this type of fractal is referred to as multi-fractal. For instance, a given time series could exhibits long term correlation at small scales $s \ll s_{x}$ and another type or even the absence of correlation at larger scale $s \gg s_{x}$. In other cases, the scaling behavior could be more complex. One of the techniques proposed in literature to perform the multi-fractal analysis of time series is the MultiFractal Detrended Fluctuation Analysis (MF-DFA) developed by [12], which is the extension of the detrended fluctuation analysis (DFA) originally proposed by [13]. Such a kind of analysis has been successful applied in the field of biomedical signals by several authors, see [14] for a review of the subject. Furthermore [14] implemented also a useful Matlab version of the code to perform MF-DFA, considered in this paper.

Given a time series $x_{i}$ of lenght $N$ with compact support, i.e. $x_{i}=0$ for an insignificant fraction of values only, the MF-DFA consists of the following steps as described in [12].

- Step 1: Determine the profile

$$
Y(i) \equiv \sum_{k=1}^{i}\left[x_{k}-\langle x\rangle\right], i=1, \ldots, N
$$

where again $\langle x\rangle$ represents the mean of given time series.

- $\quad$ Step 2: Divide the profile $Y(i)$ into $N_{s} \equiv \operatorname{int}(N / s)$ non overlapping segments of equal length $s$, starting from the leftmost sample (i.e $i=1$ ). In order not to disregards any sample of the time series, due to the fact that $\operatorname{int}(N / s) \leq(N / s)$, the original time series is divided once again, starting from the rightmost sample. Thereby, in total $2 N_{s}$ segments are obtained.

- Step 3: Calculate the local trend for each of the $2 N_{s}$ segments by a least square fit of the series and determine the variance for each segment $\nu, \nu=1, \ldots, N_{s}$ by using the formula:

$$
F^{2}(s, \nu) \equiv \frac{1}{s} \sum_{i=1}^{s}\left\{Y[(\nu-1) s+i]-y_{\nu}(i)\right\}^{2}
$$

and for $\nu, \nu=N_{s}+1, \ldots, 2 N_{s}$ by using the formula:

$$
F^{2}(s, \nu) \equiv \frac{1}{s} \sum_{i=1}^{s}\left\{Y\left[N-\left(\nu-N_{s}\right) s+i\right]-y_{\nu}(i)\right\}^{2}
$$

In expressions (8) and (9), $y_{\nu}$ represents the fitting polynomial for segment $\nu$. Such a fitting can be performed by using polynomials of various order $m$.

- Step 4: Average over all segments in order to obtain the q-order fluctuation function expressed by

$$
F_{q}(s) \equiv\left\{\frac{1}{2 N_{s}} \sum_{\nu=1}^{2 N_{s}}\left[F^{2}(s, \nu)\right]^{\frac{q}{2}}\right\}^{\frac{1}{q}}
$$


where the variable $q$ can assume any real value, except zero. For $q=2$ the MF-DFA reduces to the standard DFA. By simple calculations it can be realized that $F_{q}(s)$ is defined only for $s \geq m+2$.

- Determine the scaling behavior of the fluctuation function by analyzing the log-log plots of $F_{q}(s)$ versus $s$ for each value of $q$. If a given series $x_{k}$ is long-range power law correlated, $F_{q}$ increases for large values of $s$, as a power law, i.e. as represented by the following expression

$$
F_{q}(s) \sim s^{h(q)}
$$

In general, the exponent $h(q)$ depends on $q$. Since for stationary time series $h(2)$ is identical to the Hurst exponent, it has been referred to as the generalized Hurst exponent. For monofractal time series with compact support, $h(q)$ is independent of $q$, since the scaling behavior of the variances $F^{2}(s, \nu)$ is identical for all segments $\nu$, and the averaging procedure in Equation (10) will give identical scaling behavior for all values of $q$. Only if small and large fluctuations scale differently, there will be a significant dependence of $h(q)$ on $q$. If we consider positive values of $q$, the segments $\nu$ with large variance $F^{2}(s, \nu)$ (i. e. large deviations from the corresponding fit) will dominate the average $F_{q}(s)$. Thus, for positive values of $q, h(q)$ describes the scaling behavior of the segments with large fluctuations. Usually the large fluctuations are characterized by a smaller scaling exponent $h(q)$ for multifractal series. On the contrary, for negative values of $q$, the segments with small variance $F^{2}(s, \nu)$ will dominate the average $F_{q}(s)$. Hence, for negative values of $q, h(q)$ describes the scaling behavior of the segments with small fluctuations, which are usually characterized by a larger scaling exponent. In [12] it has also been discussed the relation between the MFDFA and the standard DFA in the case of stationary time series. In particular, it has been established the relation between the classical multifractal standard scaling exponent $\tau(q)$ and the generalized Hurst exponent as expressed by the formula:

$$
\tau(q)=q h(q)-1
$$

It is also to be observed that the generalized Hurst exponent $h(q)$ is different from the generalized multifractal dimension $D(q)$ that is sometimes considered instead of $\tau(q)$. Indeed $D(q)$ is expressed as

$$
D(q)=\frac{\tau(q)}{q-1}=\frac{q h(q)-1}{q-1}
$$

thus it is possible to observe that while $h(q)$ is independent of $q$ for monofractal time series with compact support, $D(q)$ depends on $q$.

Another way to characterize a multifractal series is to consider the singularity spectrum $f(\alpha)$, that is related to $\tau(q)$ via the Legendre transform

$$
\begin{aligned}
& \alpha=\tau^{\prime}(q) \\
& f(\alpha)=q \alpha-\tau(q)
\end{aligned}
$$

where $f(\alpha)$ is the so-called multi-fractal spectrum.

If the observed time series is a single scale fractal series, the function $f(\alpha)$ is a constant. Otherwise, if the time series is multi-fractal, it usually exhibits a bell-like shape. The larger $\alpha$ values reflect the features of small range, while the small $\alpha$ reflect the feature of large range.

In order to characterize the multifractal spectrum, the width of the spectrum is defined as $\Delta \alpha=\alpha_{\max }-\alpha_{\min }$ and the corresponding deviation of fractal dimension $\Delta f=f\left(\alpha_{\text {min }}\right)-$ $f\left(\alpha_{\max }\right)$. The width of the spectrum $\Delta \alpha$ indicates the non uniform degree of measure distribution. Generally speaking, the larger $\Delta \alpha$ indicate the more heterogeneous distribution while the smaller $\Delta \alpha$ indicates the more uniformly distributed fractal region.

$f_{\max }$ and $f_{\min }$ can describe the fractal dimension of the subsets, when the measurement values attain their minimum and maximum values respectively. Furthermore since $f^{\prime}(\alpha)=$ $q$, we have that the the maximum $f$ value, say $f_{\max }$, is obtained for $q=0$, and thus from equation (14) it results that $f_{\max }=\tau(0)$. Indicating as $d_{f}=\tau(0)$, for the subsets $f(\alpha)<f_{\max }$ all their fractal dimensions are smaller then $d_{f}$. Since $\Delta f$ may be either positive or negative, we have the following cases:

- $\Delta f>0$. In this case the largest subset is greater than the minimal one in terms of probability measures and the multifractal spectrum shows a left hook shape.

- $\Delta f<0$. In this case the largest subset is smaller than the minimal one and the multifractal spectrum shows a right hook shape.

\section{Power spectra analysis}

Power spectrum analysis has been considered to analyze fractal time series since long time ago [15]. The power spectral density function $P(f)$ shows the strength of the energy variations as a function of frequency. It is possible to obtain energy within a specific frequency range by integrating $P(f)$ within that frequency range. Computation of $P(f)$ can be done directly by the well-known FFT algorithm or by computing the Fourier transform of the autocorrelation function. When a power spectrum density $P(f)$ shows a noise behavior without an eminent peak, it is naturally approximated to follow a power law spectrum. One of the peculiarity of several geophysical signals is that they exhibits the known $1 / f^{\beta}$ noise features. Roughly speaking a $1 / f^{\beta}$ noise has three main features:

- It has an autocorrelation that decays so slowly that its sum does not converge to a finite number. Specifically, the correlation $C(k)$ is given by a power law function, $C(k)=|k|^{-\gamma}$, with $\gamma$ between 0 and $1, k$ being the lag. This means that the process is long-range dependent.

- The $\log \log$ power spectrum of a $1 / f^{\beta}$ process is linear with slope $\beta$, where $\beta$ is usually taken to range from $\beta=0.5$ to $\beta=1.5$. Note that a white noise time series has a slope of $\beta=0$, due to the fact that its energy is equally distributed for all frequencies and thus the power spectrum is flat, while a random walk (i.e. differences between consecutive samples represents a white noise) shows a slope of $\beta=2$.

- A third feature of a $1 / f^{\beta}$ process is that it is selfsimilar, i.e. the statistical properties of the time series are the same regardless of the scale of measurement, and hence the process lacks a characteristic time scale. 
Fig. 1. Estimation of the box dimension for daily (upper) and hourly (lower) wind speed recorded at Aberdeen.
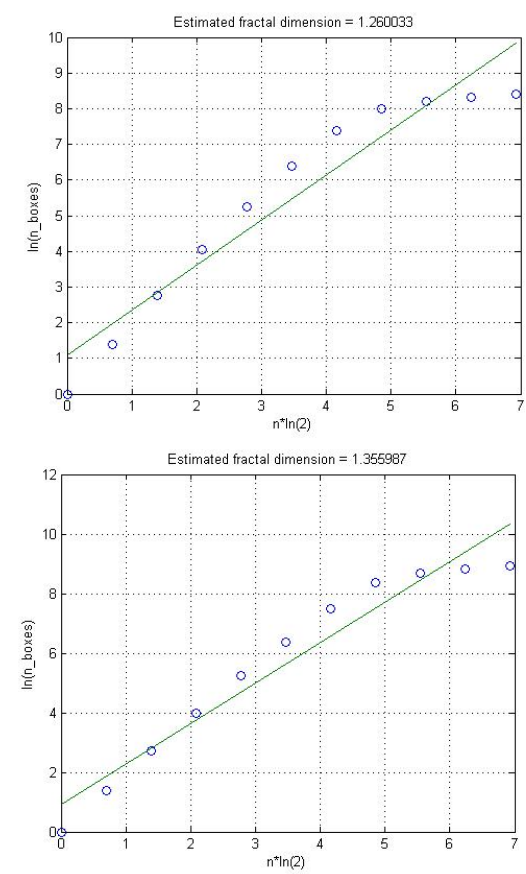

Wind speed time series exhibits similar kind of features, as it will be discussed in section III.

\section{WIND SPEED ANALYSIS}

The data sets considered in this paper consist of wind speed time series recorded in different geographic areas of both Italy and USA. Data recorded in Italy consists of time series provided the Politecnico di Milano and recorded in the city of Como and by the ARPA Piemonte, recorded at Caselle (Torino). Data recorded at Como are sampled with 5 minutes sampling time while data recorded at Caselle have been provided as daily mean values only. Wind speed time series recorded in USA have been provided by the Solar Radiation Monitoring Laboratory of the University of Oregon and specifically recorded at the stations referred to as Aberdeen, Parma, Picabo and Twinfalls, located in Idaho (USA). While the Aberdeen data sets have been provided with 10 minutes sampling time, the remaining data set has been provided as daily mean values only.

\section{A. Fractal analysis of wind speed time series}

As described in section II, fractal analysis of time series essentially consists in computing the fractal dimension $D$. This can be done directly by using the box counting algorithm or by using the relation $D=2-H$, after estimation of the Hurst exponent $H$. In this paper, both strategies have been considered in order to have independent estimations. As an example, the box dimension $D$ computed for both daily and hourly wind speed time series recorded at Aberdeen from 2000 to 2012 is shown in Figure 1.

In more detail, Figure 1 shows the log-log graph of $N_{\varepsilon}$ versus the size $\varepsilon$ for the considered time series and the computed regression line; the slope represents the estimated fractal dimension. In this paper to
TABLE I. FRACTAL DIMENSION $D$ AND HURST EXPONENT $H$ FOR DAILY MEAN WIND SPEED.

\begin{tabular}{|c|c|c|}
\hline Station & D & H \\
\hline Aberdeen & 1.26 & 0.73 \\
\hline Caselle & 1.22 & 0.82 \\
\hline Como & 1.06 & 0.90 \\
\hline Parma & 1.22 & 0.76 \\
\hline Picabo & 1.24 & 0.72 \\
\hline TwinFalls & 1.14 & 0.93 \\
\hline
\end{tabular}

perform $D$ estimation the box counting algorithm coded by B. Flemisch, IANS, University of Stuttgart, available from http: / / www. mathworks.it/matlabcentral/ has been considered. In the particular example, the estimated box counting dimension, using a number of steps equal to 10 , was $(D=1.26)$ and $(D=1.36)$ for daily and hourly mean wind speed respectively.

An example of estimation of the Hurst exponent by using the corrected Empirical and Theoretical $(R / S)$ analysis (see [11] for details) to both daily and hourly wind speed time series recorded at Aberdeen is shown in Figure 2.

Fig. 2. Estimation of the Hurst exponent by using the Empirical and corrected Theoretical (R/S) analysis for daily and hourly mean wind speed recorded at Aberdeen.
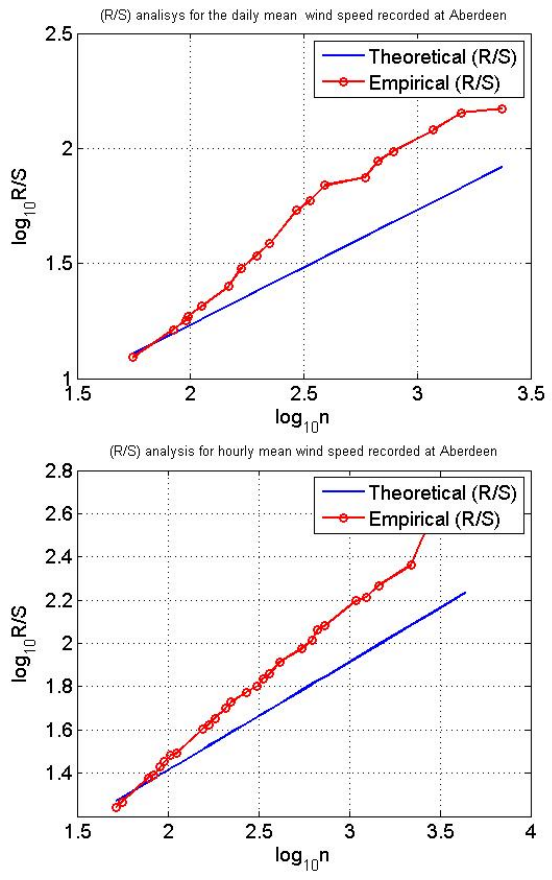

Estimated values for $D$ and $H$ for all considered recording stations are summarized in Table I and II for daily and hourly time series respectively.

From Table I it is possible to observe that the daily mean wind speed time series exhibits an average fractal dimension of about 1.19 while the average value of the Hurst exponent is about 0.81 . Thus the relation $D=2-H$ is experimentally verified. Table II shows that, at hourly scale, the average value for the box dimension is about $D=1.41$ which means simply that hourly mean time series are more irregular than the corresponding daily time series. However, in this case the relation $D=2-H$ is met with lower approximation, possibly due to the fact that only two recording stations with hourly 
TABLE II. FRACTAL DIMENSION $D$ AND HURST EXPONENT $H$ FOR HOURLY MEAN WIND SPEED.

\begin{tabular}{|c|c|c|}
\hline Station & D & H \\
\hline Aberdeen (hourly) & 1.36 & 0.73 \\
\hline Como (hourly) & 1.46 & 0.78 \\
\hline
\end{tabular}

Fig. 3. MF-DFA analysis performed on daily mean wind speed recorded at Aberdeen from 2000 to 2012.
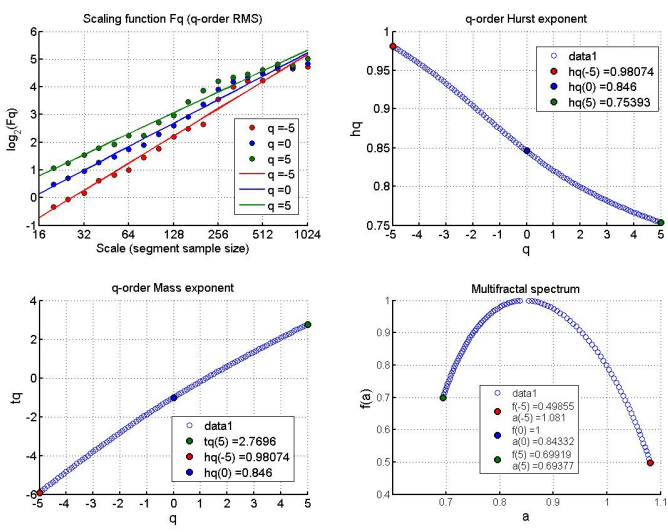

time step were considered for the estimation.

It is to be observed that time series have been recorded in different areas of both Italy and USA and thus it is reasonable to expect that similar results can be obtained for others geographical situations. Furthermore, since the values estimated for the $H$ exponent are in the range $0.5<H<1$ for all considered recording stations and time scales it is possible to conclude that wind speed time series are long-term positively correlated. In other words, a high value in the wind speed series will probably be followed by another high value and that the values a long time into the future will also tend to be high. Similarly it will occur for low wind speed values.

\section{B. Results of MF-DFA analysis}

For brevity reasons, we will detail results of MF-DFA analysis performed on daily average time series at Aberdeen only. Analysis was carried out by using the MATLAB tool developed by [14]. The output of the MF-DFA analysis for Aberdeen is shown in Figure 3, while for all stations is summarized in Table III.

The upper-leftmost Figure 3 shows the log-log plot of the scaling function $F_{q}(s)$ versus the scale $s$ for various values of the $q$ parameter. First of all it is to be observed that these trends are well approximated by straight lines, and this is decisive to say that the considered data set is scale invariant and therefore has fractal behavior. The slope of the regression line for a given value $q$ represents the generalized Hurst exponent $h(q)$. Another interesting aspect is that the regression lines differ from each other for various values of the $q$ order, thus representing a further element in favor of the hypothesis of multi-fractal nature of the considered data set. Indeed, if the regression lines were superimposed to each other for various $q$ then the conclusion would be that the data set has a mono-fractal behavior. The upper-rightmost Figure 3 shows the generalized Hurst exponent $h(q)$ versus $q$. For positive values of $q, h(q)$ describes the scaling behavior of the
TABLE III. MULTIFRACTAL FEATURES OF DAILY WIND SPEED DATA FOR EACH OBSERVATION STATION.

\begin{tabular}{|c|c|c|c|c|}
\hline station & $\Delta \alpha$ & $\Delta f$ & $d_{f}$ & $h(2)$ \\
\hline Aberdeen & 0.39 & 0.20 & 1 & 0.80 \\
\hline Caselle & 0.48 & -0.07 & 1 & 0.85 \\
\hline Como & 0.44 & 0.13 & 1 & 0.85 \\
\hline Parma & 0.52 & 0.28 & 1 & 0.86 \\
\hline Picabo & 0.59 & -0.10 & 1 & 0.83 \\
\hline Twinfalls & 0.42 & 0.20 & 1 & 0.92 \\
\hline
\end{tabular}

Fig. 4. Power spectra of daily and hourly mean wind speed recorded at Aberdeen.
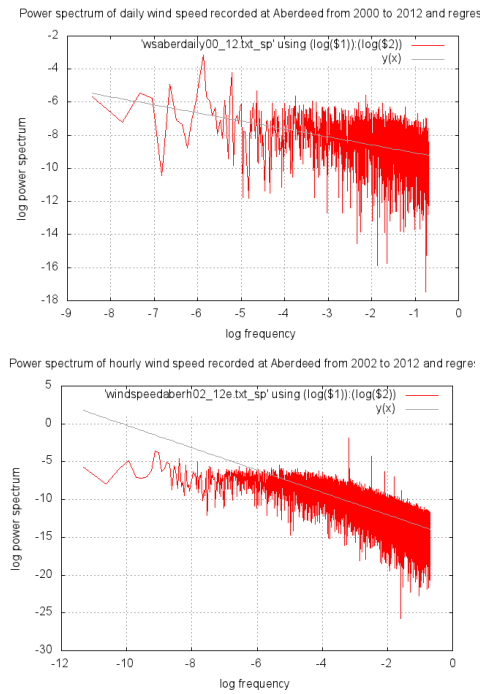

segments with large fluctuations while for negative values of $q, h(q)$ describes the scaling behavior of the segments with small fluctuations. The lower-leftmost Figure 3 represents the mass exponent $\tau(q)$ versus $q$ and finally the lower-rightmost Figure 3 represents the multifractal spectrum for the wind speed recorded at Aberdeen.

Table III shows that daily mean time series have an average width of the multifractal spectra of $\Delta \alpha=0.47$. This kind of analysis indicates a mean value of the Hurst exponent $H=h(2)=0.85$ which is a bit higher then the one obtained through the mono-fractal analysis $(H=0.81)$, but in essence it confirms the validity of results shown in the previous section III-A,

\section{Results of power spectral analysis}

The power spectra of wind speed recorded at Aberdeen at hourly and daily time scale and the corresponding regression lines are reported in Figure 4. It is possible to see that the loglog graphics are well approximated by power law. Furthermore it can be seen that the slope $\beta$ of the regression line depends on the time scale, being lower for the daily mean time series with respect to the hourly mean. $\beta$ values for all considered time series and the corresponding least square fitting standard error, $\beta_{\text {error }}$, are reported in Table IV and $\mathrm{V}$ for daily and hourly time series respectively. It is possible to observe that the average slope of power spectra assumes the value of about 0.46 and 1.37 at daily and hourly scale respectively.

As an example in Figure 5 the autocorrelation functions and approximated decaying laws for both daily and hourly 
TABLE IV. SLOPE OF THE POWER SPECTRUM AND CORRESPONDING LEAST SQUARE FITTING STANDARD ERROR COMPUTED FOR DAILY MEAN TIME SERIES.

\begin{tabular}{|c|c|c|}
\hline station & $\beta$ & $\beta_{\text {error }}$ \\
\hline Aberdeen & 0.48 & $+/-0.03$ \\
\hline Caselle & 0.44 & $+/-0.03$ \\
\hline Como & 0.61 & $+/-0.05$ \\
\hline Parma & 0.49 & $+/-0.03$ \\
\hline Picabo & 0.40 & $+/-0.03$ \\
\hline Twinfalls & 0.34 & $+/-0.05$ \\
\hline
\end{tabular}

TABLE V. SLOPE OF THE POWER SPECTRUM AND CORRESPONDING ERROR COMPUTED FOR HOURLY MEAN TIME SERIES.

\begin{tabular}{|c|c|c|}
\hline station & $\beta$ & $\beta_{\text {error }}$ \\
\hline Aberdeen & 1.48 & $+/-0.01$ \\
\hline Como & 1.26 & $+/-0.01$ \\
\hline
\end{tabular}

mean wind speed at Aberdeen are shown. It is possible to see that at daily scale the autocorrelation of wind speed decays approximately as $C(k)=|k|^{-0.8}$ while at hourly time scale as $C(k)=|k|^{-0.5}$.

Fig. 5. Autocorrelation for hourly (red line) and daily (blue line) mean wind speed recorded ad Aberdeen. The cyan and red lines represent the $|k|^{-0.8}$ and $|k|^{-0.5}$ decaying laws, respectively.

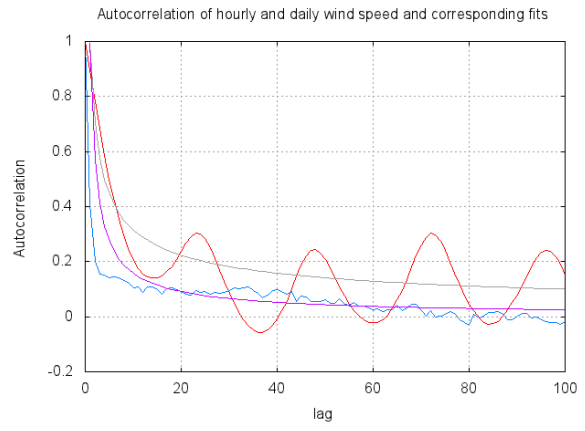

In summary, results of the spectral analysis show that wind speed can be classified as a Brown $1 / f$ noise. In particular at daily time scale wind speed looks quite similar to a white noise since its correlation function reaches a value of about 0.4 after lag 1 , while at hourly time scale it keeps some degree of correlation until lag 6.

\section{CONCLUSiON}

In this paper we have performed a fractal, multi-fractal and power spectra analysis of wind speed time series recorded at different meteorological stations. As a general comment, we can affirm that the fractal behavior of considered time series emerges clearly from all the approaches. Indeed key parameters of fractal analysis has been confirmed by several independent techniques. In more detail

- The fractal analysis shows that daily and hourly mean wind speed time series exhibit an average value of the box dimension of $D=1.19$ and $D=1.41$ respectively, while the estimated average value of the Hurst exponent is $H=0.81$ and $H=0.75$ respectively, which allow to quantifying the degree of persistency in the behavior of both daily and hourly mean wind speed.
- The multi-fractal analysis further confirms the fractal behavior and shows some features of the fractal spectra which exhibits a classical bell-like shape with average width $\Delta \alpha=0.47$.

- The power spectrum analysis points out that the considered time series behave as a $1 / f^{\beta}$ noise characterized by slope $\beta=0.46$ and $\beta=1.37$ for daily mean and hourly mean wind speed time series, respectively. In other terms it is possible to say that wind speed time series are Brown noise like.

Since the data were recorded in different geographical areas it may be reasonable to assume that the validity of such values could be extended. However, this aspect requires further investigations. Results of the performed analysis may be useful for modeling purposes. Since wind speed time series have been demonstrated to be low dimensional chaotic, it may be helpful to consider specific non linear modeling strategies.

\section{REFERENCES}

[1] A. Costa, A. Crespo, J. Navarro, G. Lizcano, H. Madsen, and E. Feitosa, "A review on the young history of the wind power short-term prediction," Renewable Sustainable Energy Reviews, vol. 12, pp. 1725-1744, 2008.

[2] G. Giebel, R. Brownsword, G. Kariniotakis, M, Denhard, and C. Draxl, "The state of the art in short-term prediction of wind power - a literature overview," Online, pp. 1-110. [Online]. Available: http://www.anemos-plus.eu

[3] T.-P. Chang, H.-H. Ko, F.-J. Liu, P.-H. Chen, Y.-P. Chang, Y.-H. Liang, H.-Y. Jang, T.-C. Lin, and Y.-H. Chen, "Fractal dimension of wind speed time series," Applied Energy, vol. 93, pp. 742-749, 2012.

[4] M. Piacquadio and A. DelaBarra, "Multifractal analysis ow wind velocity data," Energy for Suistanable Development, vol. 46, pp. 1-14, 2014.

[5] B. B. Mandelbrot, The fractal geometry of nature, Macmillan, Ed. Macmillan, 1983.

[6] S. H. Strogatz, Nonlinear dynamics and chaos: with applications to physics, biology, chemistry, and engineering, L. Perseus Books Publishing, Ed. Perseus Books Publishing, L.L.C., 1994.

[7] B. Sivakumar, "Chaos theory in geophysics: past, present and future," Chaos, Solitons and Fractals, vol. 19, pp. 441-462, 2004.

[8] N. Sarkar and B. Chaudhuri, "An efficient differential box-counting approach to compute fractal dimension of image," IEEE Trans. Syst. Man Cybernet., vol. 24, pp. 115-120, 1994.

[9] S. Buczkowski, S. Kyriacos, F. Nekka, and L. Cartilier, "The modified box-counting method : Analysis of some characteristic parameters," Pattern Recognition, vol. 4, pp. 411-418, 1998.

[10] B. Mandelbrot and J. Wallis, "Robustness of the rescaled range r/s in the measurement of noncyclic long-run statistical dependence," Water Resources Research, vol. 5, pp. 967-988, 1969.

[11] R. Weron, "Estimating long range dependence finite sample properties and confidence intervals," Physica A, vol. 312, pp. 285-299, 2002.

[12] J. W. Kantelhardt, S. A. Zschiegner, E. Koscielny-Bunde, A. Bunde, S. Havlin, and H. E. Stanley, "Multifractal detrended fluctuation analysis of nonstationary time series," Physica A, vol. 316, pp. 87-114, 2002.

[13] C. Peng, S. V. Buldyrev, S. Havlin, M. Simons, H. E. Stanley, and A. L. Goldberger, "Mosaic organization of dna nucleotides," Phys. Rev. E, vol. 49, pp. 1-14, 1994.

[14] E. A. F. Ihlen, "Introduction to multifractal detrended fluctuation analysis in matlab," Frontiers in Physiology, pp. 1-18, 2012.

[15] T. Higuchi, "Relation between the fractal dimension and the power law index for a time series- a numerical investigation," Physica D, vol. 46, pp. 254-264, 1990. 